\title{
Electron spectroscopy using two-dimensional electron detection and a camera in a single electron counting mode
}

\author{
M. Vos, ${ }^{\text {a) }}$ M. R. Went, and E. Weigold \\ Atomic and Molecular Physics Laboratories, Research School of Physics and Engineering, \\ Australian National University, Canberra, Australian Capital Territory 0200, Australia
}

(Received 5 March 2009; accepted 18 May 2009; published online 10 June 2009)

\begin{abstract}
A brief description is given of an economical implementation of the read out of a two-dimensional detector in an electron spectrometer by a charge coupled device camera, using a pulse counting mode. Count rates up to $10 \mathrm{kHz}$ can be handled in this way. A comparison with results obtained using a resistive anode detector is given for the case of electron scattering from Xe atoms. Good agreement was obtained between both detection techniques, establishing the validity of the method described here. (C) 2009 American Institute of Physics. [DOI: 10.1063/1.3152366]
\end{abstract}

\section{INTRODUCTION}

In modern electron spectroscopy two-dimensional detectors are often essential to obtain good-quality signal in a reasonable amount of time. These detectors use microchannel plates for amplification of the detected electron. The position of the resulting charge cloud is then read out by either a resistive anode, delay-line analyzer, or a phosphor screen camera combination. The first two methods depend on small electrical signals being coupled out from the detector to the detection electronics. This requires several electrical feedthroughs and decoupling capacitors that are not really desirable in UHV. Especially for high-energy electron spectroscopies this is a significant disadvantage as arcing, if it occurs, tends to destroy the sensitive electronics. The read out via a phosphor screen and camera does not suffer from this problem. This idea is quite old (see, e.g., Refs. 1 and 2), but has recently become much more attractive with the increasing performance of digital cameras and computer hardware.

Digital cameras can be operated in two different ways as follows.

(a) By choosing a long exposure the image that builds up is the final result. The light intensity at a certain pixel is proportional to the number of electrons hitting the phosphor screen at that point. Here pixel depth (ratio of the strongest and weakest signal that can be measured by a pixel) is crucial, as it determines the ratio of the strongest and weakest feature that can be detected simultaneously. See, e.g., Ref. 3 for an example.

(b) By choosing a short exposure one can have no or rare overlap between the light emitted from separate events. Analysis of the image can then be used to reconstruct where the charge cloud of a single detected electrons hits the screen. Here the data transfer rate between the camera and computer as well as the time required by the computer to analyze the images are crucial, as it determines the number of exposures that can be measured per second and hence the maximum count rate

\footnotetext{
${ }^{a)}$ Electronic mail: maarten.vos@anu.edu.au.
}

that is allowed before spatial overlap between different events in a single exposure becomes an issue.

For our application, where we measure the (intense) elastic peak and a weak energy loss signal, a huge dynamic range is required, hence the second option is the most desirable. This method was implemented, and is described here in some detail. We describe here the use of an AVT PIKE F-032B camera that communicates with the computer using a FireWire S800 (IEEE 1394b) protocol. For completeness we describe the electron optics part of the spectrometer in some detail. Previous results of the spectrometer, using a resistive anode (Quantar Technology) have been published, ${ }^{4-6}$ and a comparison of spectra obtained in both ways is made.

\section{APPARATUS}

The electron optics part of our gas-phase high-angle electron scattering spectrometer is shown in Fig. 1. It consists of a five element slit lens followed by a hemispherical analyzer operating at a $200 \mathrm{eV}$ pass energy with at the exit (behind a grid) a $40 \mathrm{~mm}$ effective diameter multichannelplate pair. Thus the vertical coordinate of the impact is determined by the position where the electron entered the slit lens, the horizontal coordinate is determined by the electron energy. For the gap distance between inner and outer hemisphere of $30 \mathrm{~mm}$ we measure (at $200 \mathrm{eV}$ pass energy) simultaneously an energy window of $25 \mathrm{eV}$. The energy resolution of the spectrometer is mainly determined by the beam spread of the incoming electron beam. The electron gun has a $\mathrm{BaO}$ cathode for low thermal spread, and the energy resolution of the spectrometer (at moderate output current of the gun) is $0.5 \mathrm{eV}$ full width at half maximum. Thus a modest spatial resolution of 1:100 or better is required for the read out to have negligible influence on the performance of the spectrometer. We choose a camera with a VGA resolution (640 $\times 480)$, but that can operate at a high frame rate $(\approx 200$ frames/sec). The part of the phosphor screen that is illuminated is rectangular, with the shorter dimension corresponding to the energy-dispersive direction. The camera is oriented accordingly. 

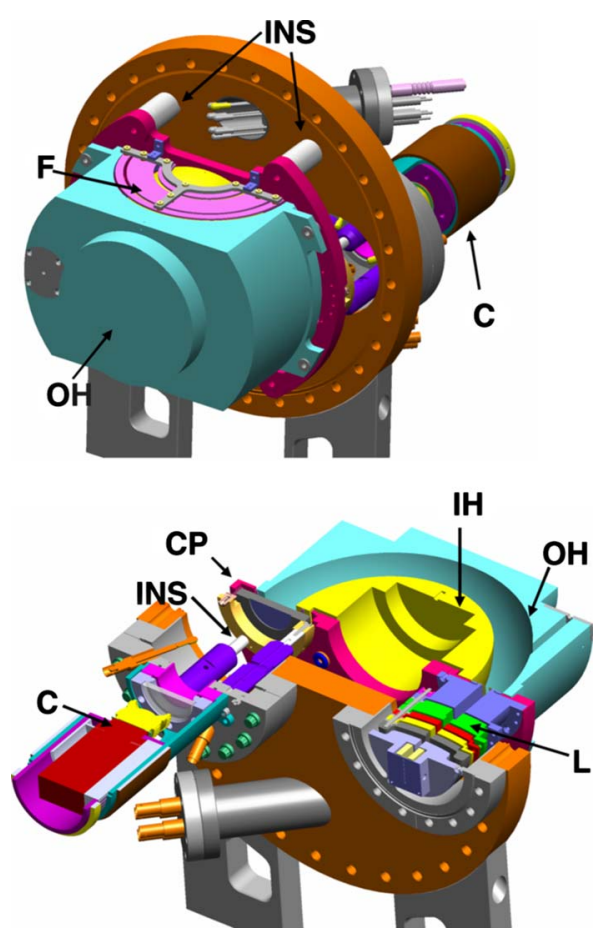

FIG. 1. (Color online) Front and back view of the electron optics, mounted on a 14 in. conflat flange. It shows the inner and outer hemisphere (IH and $\mathrm{OH}$ ) slit lenses (L), camera (C), channel plate/phosphor assembly (CP), high-voltage insulators (INS), and fringe field correctors (F).

The detector used was purchased from El-Mul (multichannel plate assembly model C050VP), with a P47 Scintimax phosphor screen. The phosphor screen is biased by 3 $\mathrm{keV}$ relative to the back channelplate. The decay time of this phosphor $(120 \mathrm{~ns})$ is short enough to ensure that no event will contribute to two different exposures, and the yield of 25 photons for a $3 \mathrm{keV}$ electron hitting the screen is sufficient to ensure adequate light intensity in the camera. The distance between the phosphor screen and the exit window (standard $23 / 4$ in. conflat viewport) was $135 \mathrm{~mm}$. The camera with a lens with $12 \mathrm{~mm}$ focal distance (aperture size used was $\mathrm{f} / 2.8$ ) was positioned close to this window. The detector assembly is mounted and electrically connected on a 6 in. conflat flange, and can be removed without opening the main (14 in.) flange. The electrical connections are made by coaxial connectors [SHV10 from Insulator Seal (ISI)] for detector and main hemisphere voltages, and a seven-pin power boot connector (also from ISI) for the lens elements and fringe field correctors.

For completeness we give a short overview of the electronics used in this spectrometer. It is sketched in Fig. 2. We use a commercial electron gun power supply (Bertan, EB30A, modified such that the maximum output voltage is limited to $10 \mathrm{keV}$ ). This power supply is also used as the main high voltage source for the detector electronics. A small (500 max) offset power supply (EMCO CA series) is used to define the mean analyzer (base plate) voltage relative to the gun high voltage. This, and the other power supplies floating at the base plate potential are powered via a dc-to-dc converter (Ultravolt 15FL12-12W-i/o), which provides up to 15 $\mathrm{kV}$ isolation. This unit also has a $0-5 \mathrm{~V}$ analog input (relative to ground) that result in a $0-5 \mathrm{~V}$ output, relative to the high voltage side of the converter. This voltage is used to control the floating power supply that provides the offset voltage under computer control. Three other EMCO CA units (outputs of $0-2 \mathrm{keV}$ ) supply the lens voltages and an additional 2 EMCO CA series (500 V max, one positive and one negative output) provides the potential for the inner and outer hemisphere. The lens voltages and hemisphere voltages are constant during an experiment and are set using a (floating) resistive divider, manually controlled using perspex rods.

The high voltage to the channel plate and the phosphor screen are supplied by two $3 \mathrm{keV}$ units (Spellman High Voltage model MCP). These units have output insulation of up to $16 \mathrm{keV}$ with controls at ground potential. This allows these units to supply the $3 \mathrm{keV}$ relative to the float voltage (for the channel plates) and relative to the positive channel plate output (for the phosphor screen).

The camera has a pixel depth of 14-bit, but is operated in an 8-bit mode to maximize the frame rate. The camera allows for the transfer of only a part of the $640 \times 480$ images. A reduction in the second coordinate makes higher frame rates possible. In our specific application the exposed part of the phosphor is contained within a $640 \times 430$ area. The number of frames per second obtained in practice are shown in

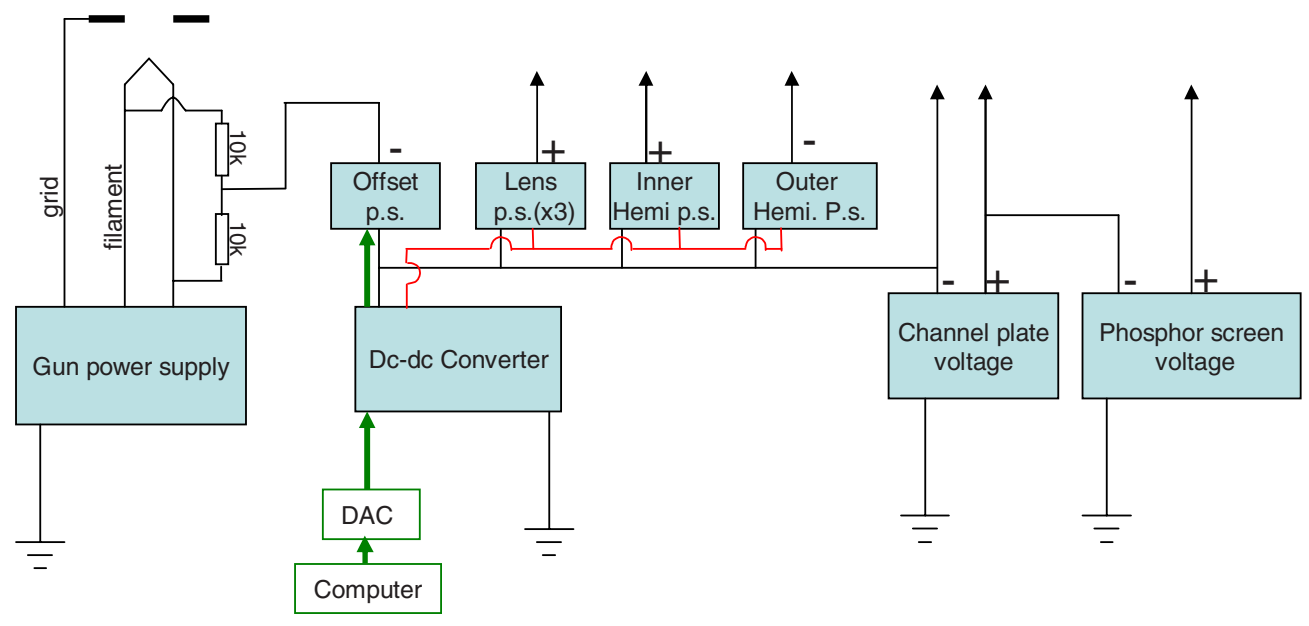

FIG. 2. (Color online) A schematic view of the spectrometer electronics. 
TABLE I. The relation between shutter time and actual obtained frame rate. To maximize frame rate the image was restricted to a $430 \times 640$ pixels out of the maximum of $480 \times 640$ pixels.

\begin{tabular}{cc}
\hline \hline $\begin{array}{c}\text { Shutter time } \\
(\mathrm{ms})\end{array}$ & Frames/s \\
\hline 3.7 & 229 \\
4.2 & 229 \\
4.6 & 215 \\
5.1 & 195 \\
\hline \hline
\end{tabular}

Table I for different exposure times. Using an exposure time of $5.1 \mathrm{~ms}$, the camera is never idle, but the frame rate is less than maximum, increasing the chance that events overlap. For shorter exposure time $(4.2$ and $3.7 \mathrm{~ms})$ the number of frames obtained is determined by the maximum transfer rate, and the camera will be part of the time idle. Thus exposure times of 4.2 or $4.6 \mathrm{~ms}$ are close to optimum.

The camera is interfaced using $\mathrm{C}++$ code and the factory-supplied FireGrab library. The images are transferred to the computer under direct memory access. When a frame is ready to be analyzed it is first scanned for those channels that are above a threshold level (usually taken between 25 and 50). If this is the case and content of neither the next array element nor the previous array element (corresponding to adjacent pixels) is larger than the current pixel coordinate, then the current pixel coordinate is identified as a possible peak and its position and content is stored. It is possible that pixels very close to the marked peak candidate are higher, and are thus more likely to correspond to the maximum intensity (center) of the event. Hence, the software checks next if any of the other peak candidates is within a user-defined distance of the current peak candidate. If so, the peak candidate with the lowest intensity is discarded. The remaining events correspond to the detected electron events. Finally, for each detected electron event we refine the impact position by determining the center-of-mass of the intensity in a userdefined area around the maximum. The precision with which the $(x, y)$ coordinate of the event is determined is less than a pixel. In the energy direction one pixel corresponds to 0.06 $\mathrm{eV}$. Thus the read-out precision is more than adequate for our experimental resolution of $0.5 \mathrm{eV}$. The resulting $(x, y)$ coordinate of the event has to be transformed in the physically more meaningful energy-angle coordinate. This is done using a calibration procedure as described by Caprari and coworkers. ${ }^{7,8}$ Finally the content of the corresponding channel of the energy spectrum and angular distribution is incremented by 1 .

Using a PC with an Intel Core 2 Duo processor running at $3 \mathrm{GHZ}$, there is enough computational power to handle count rates up to several 100 events per frame. At 40 events per frame, 200 frames a second (i.e., $8 \mathrm{kHz}$ count rate) one of the two processors is loaded by $50 \%$. Display of the spectra or detector images can be updated every few seconds as well.

For each event the computer calculates the ratio of the content of the channel with highest intensity and the summed content of a (user selectable) $3 \times 3,5 \times 5$, or $7 \times 7$ area centered on the peak. This value is a measure of the sharpness of the peak. The average of this ratio of all the events detected

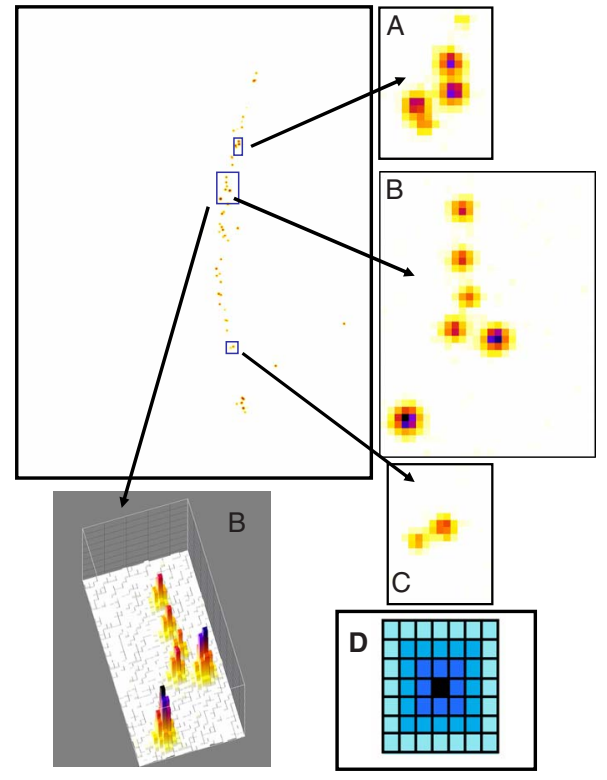

FIG. 3. (Color online) A high beam current shot of the camera showing the Xe elastic peak. The counts are shown along a vertical band, corresponding to the incoming beam energy. Characteristic region $\mathrm{A}, \mathrm{B}$, and $\mathrm{C}$, are displayed enlarged on the right. Region $\mathrm{B}$ is also plotted as a 3D plot (bottom left). In $\mathrm{D}$ we show the $3 \times 3,5 \times 5$, and $7 \times 7$ pixel area around a pixel with a maximum intensity (black) that are used in the analyzing procedure.

since the previous display update is calculated and displayed. As this average corresponds to thousands of events it is very reproducible. In this way the optimum settings of the focus and aperture of the camera lens, as well as the MCP voltage, can be determined by maximizing this sharpness value. Good results were obtained with low MCP voltages of $1.3-1.4 \mathrm{keV}$, a voltage that is $500 \mathrm{eV}$ les than in our resistive anode setup.

\section{RESULTS}

We use Xe for our test measurements as it has, due to its high $Z$ number, a large elastic scattering cross section and hence high count rates. $\mathrm{Xe}$ is introduced in the vacuum chamber through a $1 \mathrm{~mm}$ diameter needle, just above the electron beam. A measurement generally consists of a signal run (with the Xe entering through the needle) and a background run (with the Xe entering the vacuum chamber away from the interaction region. The final result is the difference of the signal and background run, hence eliminating counts due to interaction with the background gas. For details see Refs. 4 and 6

Let us first consider the case when the spectrometer is tuned to the elastic peak. In Fig. 3 we show a typical single shot of the signal run (exposure time of $4.6 \mathrm{~ms}$ ) taken under these conditions. Dots with higher intensity are mainly concentrated along a slightly curved vertical line. This corresponds to electrons that have scattered elastically from Xe, with different azimuthal angles and hence enter the slit lens at different vertical position. Thus in these plots the horizontal direction is (in first order) the dispersive direction, and the vertical direction corresponds to the azimuthal angle of the events.

In the 8-bit mode used the intensity can range from 0 to 255. The background level varied between 0 and 4 . Using a 


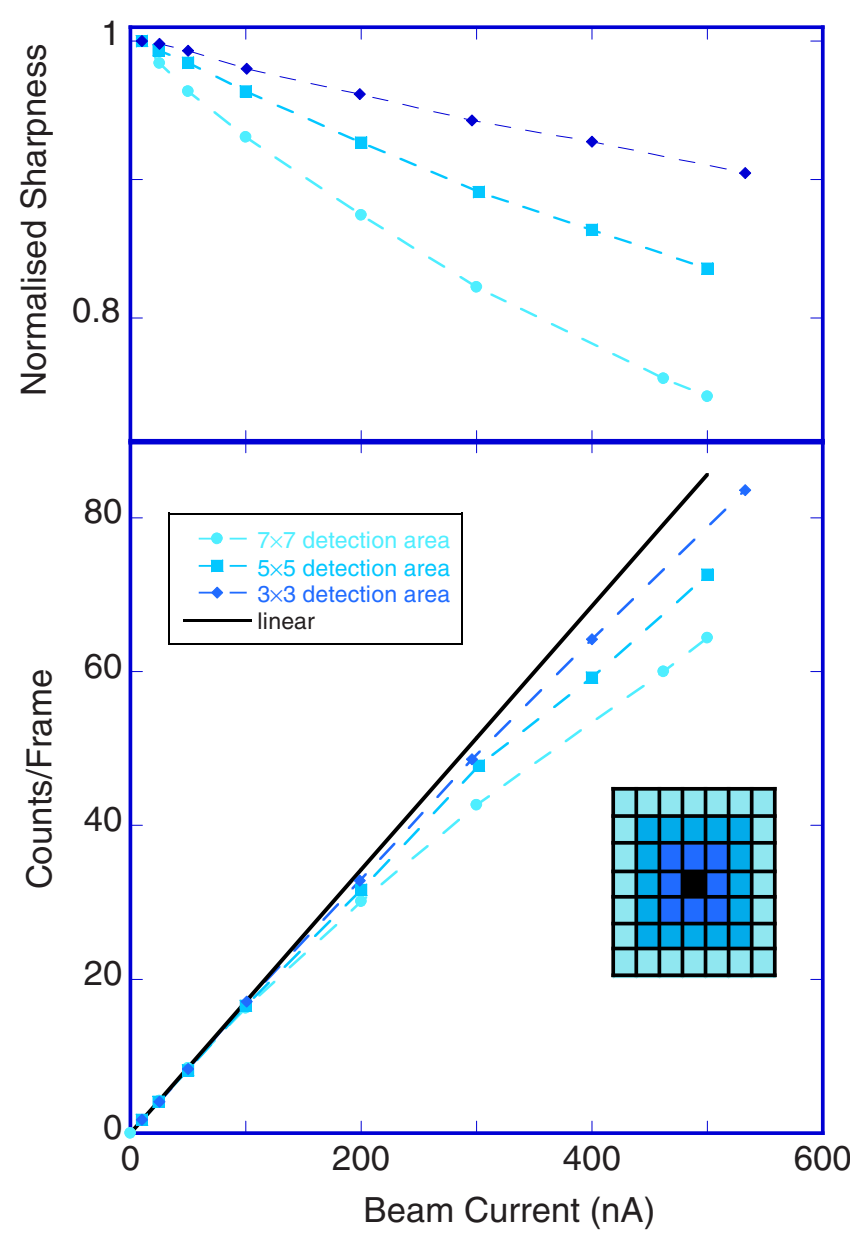

FIG. 4. (Color online) The measured "sharpness" of the events and the number of events per frames as determined for different output of the electron gun. The measurements were done using the different detection areas shown as an insert in the lower panel.

threshold value of 40 the computer identified 41 peaks in this shot, with an average peak height of 101 , and a maximum peak height of 170. Some examples of the more interesting regions are magnified as well in Fig. 3(A-C). In region A the lower-left peak is strongly asymmetric, indicating the presence of a second, somewhat weaker event at the lower right side of the main peak. As the weaker peak does not have a local maximum, it is not identified in the simple analysis procedure described here. The two peaks above each other, slightly to the right (still enlargement A) are picked up as separate peaks by the program. However, if a larger peak width is used in the analysis program the center-of-mass is influenced by the neighboring peak. Hence a smaller peak width performs best here.

Region B has six peaks, all are correctly identified by the program, for all peak widths assumed. In region $\mathrm{C}$ one detects two peaks, while using a $3 \times 3$ or $5 \times 5$ peak area, but only one peak in case of a $7 \times 7$ peak area.

We also measured the count rate as a function of electron beam energy with the elastic peak in the detection window, just as in Fig. 3. If overlap between different events is indeed happening for a significant fraction at high beam currents, then this will be evident from a slower than linear increase in events per frame with increasing incoming beam current.

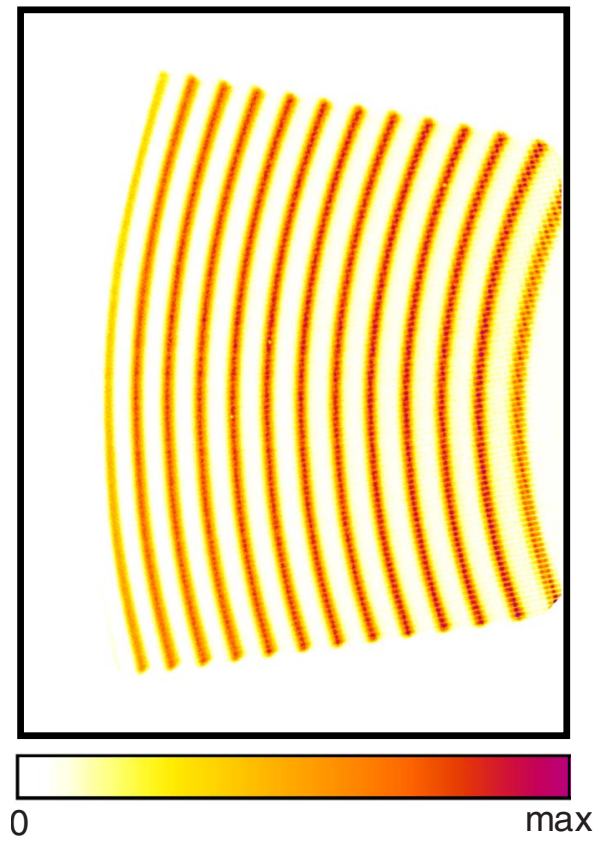

FIG. 5. (Color online) The intensity distribution as obtained by scanning the offset voltage in $2 \mathrm{eV}$ steps.

These measurements were done for the different peak detection areas, mentioned before. The results are shown in Fig. 4. Indeed at low beam currents $(<50 \mathrm{nA})$ and corresponding low number of events per frame, the count rate increases almost linearly with beam current, but at higher beam currents substantial deviations are found. These deviations are biggest if a large peak detection area is used. At $500 \mathrm{nA}$ the count rate is expected to be 85 counts/per frame (based on the observed count rate at low beam current), but the actual rate is $25 \%$ less when using the largest $7 \times 7$ peak area, and about $7 \%$ less when using the $3 \times 3$ detection area. At the same time the average sharpness of the events decrease somewhat with increasing beam current (see top panel of Fig. 4). This is plotted in the top panel, with the sharpness at lowest beam current normalized to 1 . This decrease in sharpness with increasing beam current is again strongest when using the large detection area. This is what is expected if, at large beam current, a significant number of detected events have intensity in the detection area that is due to two or more actual events.

A second matrix, also dimensioned $480 \times 640$, contains the number of times a count was detected in a certain pixel for all the exposures analyzed in the current measurement. An example of this event-count matrix is shown in Fig. 5. In this measurement we scanned the offset voltage in $2 \mathrm{~V}$ steps. At each offset voltage we acquired data for $20 \mathrm{~s}$, i.e., several thousands of exposures, and the scan was repeated several times. The image hence shows bands that are $2 \mathrm{eV}$ apart, each band correspond to the position of the elastic peak at the detector for a certain offset voltage. Note here the homogeneous intensity of the bands. This is due to the fact that the threshold level is so much lower than the average peak height, and local variations in channel plate gain have thus no influence on the final number of counts measured. Such a homogeneous response is hard to obtain with a resistive an- 


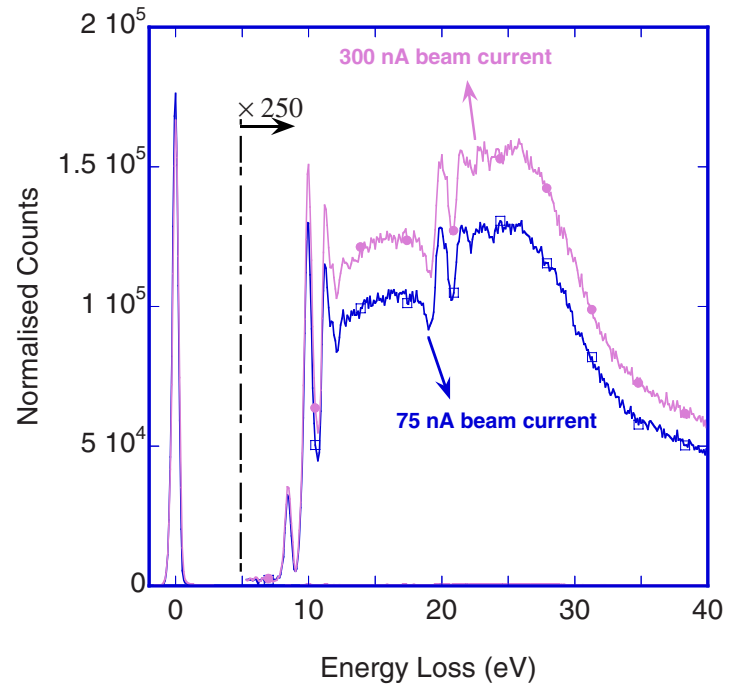

FIG. 6. (Color online) Spectra of Xe taken at $600 \mathrm{eV}$ at different beam current, and using a $7 \times 7$ detection area.

ode. Note that in a single shot (as in Fig. 3) the noise level is about 50 times lower than the maximum intensity. In the event-count matrix the maximum-to-minimum intensity ratio level can be many orders of magnitude better, provided that the threshold level for event detection is set well above the noise level. The range of possible intensities in this second matrix is of course not limited by the pixel depth of the camera and the intensity increases with the numbers of shots taken during the measurement.

The system was further tested by measuring an energy loss spectrum of Xe over a large energy range, by scanning the offset voltage in small steps $(0.5 \mathrm{eV})$ over the detector. For each event the program calculates, from the combination of offset voltage and detection coordinate, the energy loss of the scattered electron. Under these large-angle scattering conditions there exists a small intensity at larger energy loss values $^{5}$ with well-defined sharp structures. Resolving these structures, in the presence of the huge elastic peak is a challenging test of the detection method. A scattering angle of $45^{\circ}$ was used, see Ref. 5 for more background on this type of measurement. The results were normalized in such a way that there are $10^{6}$ counts in the elastic peak and are displayed in Fig. 6. Two measurements were done using 75 and $300 \mathrm{nA}$ beam current, and a $7 \times 7$ detection area. The elastic peak of the low beam current measurement is slightly narrower due to the increase in energy spread of the gun with increasing output. At an energy loss value of 5-8 eV the intensity has dropped by a factor of $2 \times 10^{4}$ from the maximum intensity of the elastic peak, underlining the dynamic range of this detection method. At $8.5 \mathrm{eV}$ energy loss we detect again a small peak followed by several more peaks and a continuum at larger energy loss values. This is due to electrons that have scattered elastically from a Xe atom, and created an electronic excitation at that atom during the scattering process. ${ }^{5}$ Note however that the intensity of the loss structures seems to depend on the beam current. This is a consequence of the normalization procedure followed and we explain this pitfall next.

For the high beam current measurement (in combination

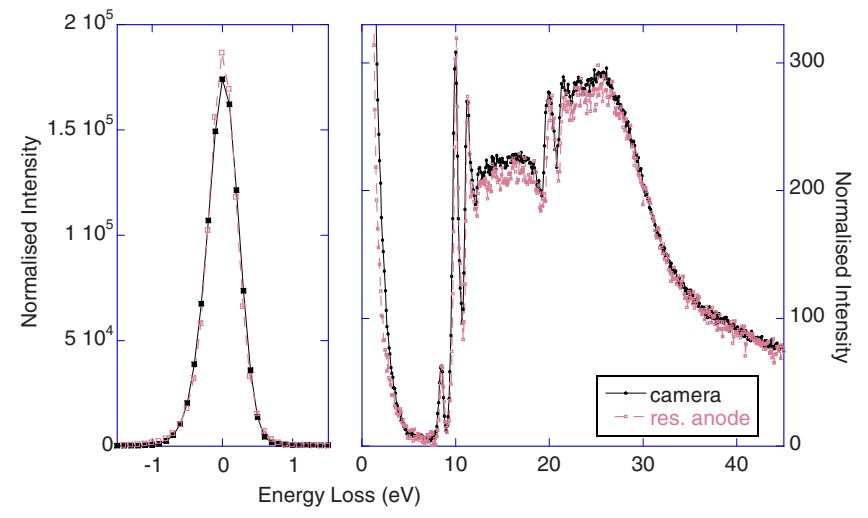

FIG. 7. (Color online) A comparison of the result for $1 \mathrm{keV}$ electron scattering from $\mathrm{Xe}$, obtained with either a camera or a resistive anode.

with the large peak detection area used) a significant fraction of elastic peak events is too close to another event to be separately distinguished. Thus the elastic peak intensity in the high beam current measurement is too low. At the position of the detector corresponding to nonzero energy loss the count rate is at least 250 times lower, and hence peak overlap is a rare occurrence. Normalizing the two measurements to the same elastic peak area results thus in the intensity of the energy loss part of the high beam current measurement being too high. The difference between both measurements are in line with the deviation from linearity shown in Fig. 4.

Using a narrow detection area $(1 \times 1)$, and a modest count rate of 25 events per frame for the elastic peak, we compared measurements taken at $1 \mathrm{keV}$ with the camera with that obtained with the resistive anode (Fig. 7). ${ }^{5}$ The elastic peak width of both detection methods are virtually the same, which is expected as the electron beam spread is the main cause of the experimental resolution. The intensity at larger energy loss values are almost the same, and nonlinearity effects under these conditions are of the order of a few percent only. The level of statistics obtained with the camera is better than for the resistive anode, suggesting a lower detection efficiency in the latter case.

\section{CONCLUSION}

Here we have shown an economical way of constructing a two-dimensional detector based on a charge coupled device camera and channel-plate phosphor assembly. The detector part (camera, MCP assembly, MCP, and phosphor power supplies, feedthroughs, and insulators) cost around $10 \mathrm{~K}$ US\$. This is considerably cheaper than the resistive anode detector it replaces (requiring resistive anode MCP assembly, preamplifiers, spectroscopic amplifiers, timing electronics, NIM-bin power supply, simultaneous sample, and hold analog-to-digital converter). In our case, with the maximum intensity concentrated at a narrow energy range corresponding to the elastic peak, it works well up to count rates of 5-10 kHz. This is somewhat less than the resistive anode can handle. If the count rate is more evenly divided over the channel plate, then it is expected that this can be safely increased tenfold and becomes comparable to the maximum 
count rate of the resistive anode. A delay-line detector is superior to either the camera or the resistive anode in terms of maximum count rate.

For high-energy spectroscopies the cross sections are usually small and count rate considerations are often secondary. Here the main advantage of the camera is the absence of any sensitive electronics that is connected (decoupled by capacitors) to the high-voltage detector. Arcing in the vacuum chamber causes rapid potential fluctuations that are transmitted through the capacitors and have the potential of damaging the electronics. Thus the setup described here makes higher energy spectrometers feasible.

The count rate restrictions are currently determined by the maximum frame rate that can be transferred to the computer. Here, improvements are expected in the medium term, when USB 3.0 and the next generation Firewire cameras become available.

\section{ACKNOWLEGMENTS}

This research was made possible by a grant of the Australian Research Council. The authors want to thank Stephen Battisson and Ron Cruikshank for technical support, and Rob Dall for stimulating discussions.

${ }^{1}$ S. P. Weeks, J. E. Rowe, S. B. Christman, and E. E. Chaban, Rev. Sci. Instrum. 50, 1249 (1979).

${ }^{2}$ P. J. Hicks, S. Daviel, B. Wallbank, and J. Comer, J. Phys. E 13, 713 (1980).

${ }^{3}$ L. Broekman, A. Tadich, E. Huwald, J. Riley, R. Leckey, T. Seyller, K. Emtsev, and L. Ley, J. Electron Spectrosc. Relat. Phenom. 144, 1001 (2005).

${ }^{4}$ M. Vos, M. Went, G. Cooper, and C. Chatzidimitriou-Dreismann, J. Phys. B 41, 135204 (2008).

${ }^{5}$ M. Went and M. Vos, J. Electron Spectrosc. Relat. Phenom. 169, 35 (2009).

${ }^{6}$ M. Vos and M. Went, J. Phys. B 42, 065204 (2009).

${ }^{7}$ R. S. Caprari, Comput. Phys. 7, 336 (1993).

${ }^{8}$ P. Storer, R. S. Caprari, S. A. C. Clark, M. Vos, and E. Weigold, Rev. Sci. Instrum. 65, 2214 (1994). 